\title{
Analysis of Bearing Faulty Cage using Non-Intrusive Condition Monitoring Techniques
}

\author{
Muhammad Irfan ${ }^{1}$, Alwadie. A ${ }^{2}$, Nordin Saad ${ }^{3}$, Muhammad Aman Sheikh ${ }^{4}$ \\ ${ }^{1,2}$ College of Engineering, Electrical Engineering Department, Najran University \\ King Abdulaziz Road, Najran, Saudi Arabia \\ ${ }^{3,4}$ Electrical and Electronic Engineering Department, Universiti Teknologi PETRONAS \\ Bandar Seri Iskandar, Tronoh, Malaysia \\ alwadie@ hotmail.com²
}

\begin{abstract}
The cage is an important element of the bearing. The cage is used to support the bearing balls and it maintains the fix distance between rotating balls. The defected cage could create defects in the balls and race ways of the bearing. It could lead to premature failure of the bearing which causes machine down time, production loss and huge maintenance cost. Traditionally, periodic checks of the bearing health are performed in industry but they did not avoid the bearing cage failures. Thus, it is essential to develop some real-time health monitoring system to analyze the status of bearing cage. Recent research developments on the bearing fault diagnosis have that non-intrusive stator current and power analysis techniques are most economical and simple as compared to intrusive vibration analysis technique. However, a limited research has been conducted to check and confirm the effectivity of stator current and power analysis techniques for the diagnosis of cage faults. Thus, the aim of this paper is to analyze cage faults using non-intrusive stator current and power analysis. The comparison of both techniques have been performed experimentally and it has been concluded that power analysis is a most suitable technique to diagnose cage faults.
\end{abstract}

Keywords: Cage Faults, Non-Intrusive Condition Monitoring, Harmonics Analysis, LabVIEW based Signal Processing.

\section{Introduction}

Cage is used to maintain a fix distance between balls of the bearing. The load applied on the shaft is transformed to the bearing balls. If balls are positioned at an equal distance then the load will be equally transformed to all balls. The balls of the bearing rotates between race ways [1-5]. Thus, to maintain the uniform motion of the shaft and to distribute the shaft load equally among all balls, the cage plays an important role. The failure of the cage will cause failure of race ways and eventually failure of the bearing [6-8]. The health of cage could be estimated through periodic checks but it could not guaranties the trouble free operation of the bearing and unexpected breakdown could occur.

The conventional vibration analysis method could be opted for continuous health monitoring of various elements of the bearing. However, expertise for complex data analysis and high cost of the vibration sensors is the limitation of the vibration analysis technique [9-12].

In a recent years, researchers have developed economical condition monitoring techniques whose data interpretation is simple and easy. The motor current analysis and power analysis are among the methods known as non-intrusive and economical ways for fault diagnosis of the bearing [13, 14].

The Hilbert transform was used by the [15] to analyze the single point defects in bearing race ways. The simulated and experimental results indicate that Hilbert transform has the ability to diagnose faults in race ways. A hybrid approach was adopted by [16] to analyze the eccentric and bearing faults. The data was collected through multi-sensors like vibration sensor, acoustic sensor and current sensor and analyzed using HilbertHuang transform. They recorded the true detection rate of approximately $99.9 \%$. The envelop analysis method was used by [17] to diagnose the bearing local faults in the presence of external vibration. The external vibration was simulated using the vibration shaker. In an extended work [18] studied the multiple local faults in bearing using vibration monitoring technique. They concluded that frequency domain analysis provides same frequency information for single fault and multiple faults. However, the amplitude has been observed to be increased in multiple local faults. Motor current analysis was used by [19] to analyze bearing faults using Fast Fourier Transform (FFT) and wavelet transform. Power analysis 
approach was used to analyze faults in bearing race ways and it was concluded that power analysis approach has the capability to diagnose these type of faults [20-26].

It has been observed from the literature that motor current analysis and power analysis approaches have the capability to diagnose the faults in raceways of the bearing. However, detailed analysis of cage faults and roller faults are not found in the published research. Thus, the scope of this paper is to analyze cage faults using motor current analysis and power analysis approaches and to compare the fault diagnosing capability of both approaches.

\section{Design of Laboratory Set-Up}

A laboratory set-up has been developed for the implementation of motor current analysis and power analysis techniques. The schematic diagram of the developed set-up has been shown in Figure 1. The mechanical parts of the test rig consist of a $3-$ phase induction motor and steel shaft coupled with motor shaft via flexible coupling. The coupling has rubber jaw to reduce the vibration occurring in the motor. The mechanical load is mounted on the steel shaft between two brackets. Two motors of the same specifications are used during the experiments. One motor has a bearing with undamaged cage while the other one has artificially defected cage. The geometry of the bearing has been described in Figure 1. The connections of the motor are in star connected for 412VAC supply. An AC variable frequency drive (VFD) is used to run the motor at various speeds. The speed of the motor is measured by Prova digital tachometer.

Loads are considered as one of the most significant factors influencing the lifetime and characteristics of the bearings. In this work, tests were performed on un-loaded and loaded conditions of the motor. The three round steel plates were mounted on a steel shaft coupled with a motor shaft to create the radial load. Aluminum coated steel comprised the round plates. The design of round plates is shown in Figure 1.

The data acquisition and processing system used in this work consists of National Instruments data acquisition card NI 6281, an AC current and voltage transformer and LabVIEW software. The bearing cage produce cage frequency which appears in motor current spectrum and power spectrum. The location of these frequencies are identified using the mathematical equations [26, 27] and are shown in Table I and II.

Table I. Cage Frequencies in Motor Current Spectrum

\begin{tabular}{|c|c|c|}
\hline Motor Load & Motor Speed (RPM) & Cage Frequencies (Hz) \\
\hline No Load & 1485 & $40.60,59.40$ \\
\hline Medium Load & 1445 & $40.80,59.20$ \\
\hline Full Load & 1405 & $41.10,58.90$ \\
\hline
\end{tabular}

Table II. Cage Frequencies in Motor Power Spectrum

\begin{tabular}{|c|c|c|}
\hline Motor Load & $\begin{array}{c}\text { Motor Speed } \\
(\mathrm{RPM})\end{array}$ & Cage Frequencies (Hz) \\
\hline No Load & 1485 & $90.60,109.40$ \\
\hline Medium Load & 1445 & $90.80,109.20$ \\
\hline Full Load & 1405 & $91.10,108.90$ \\
\hline
\end{tabular}

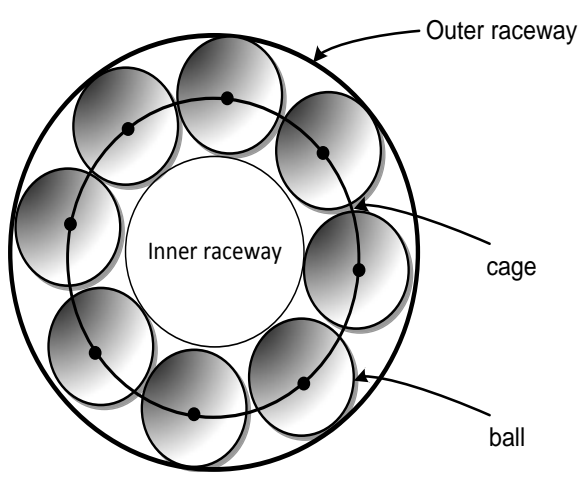

(a)

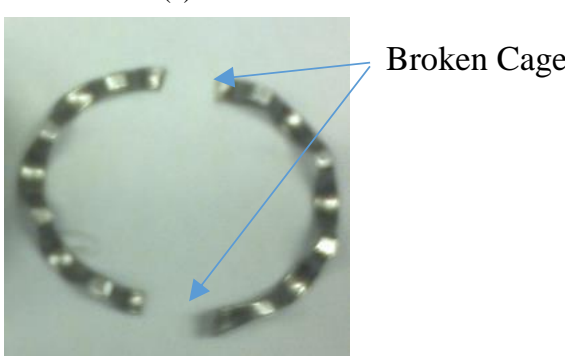


(b)

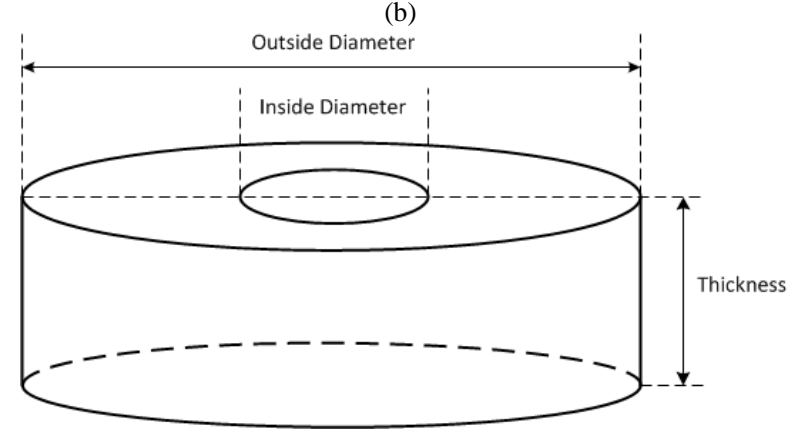

(c)

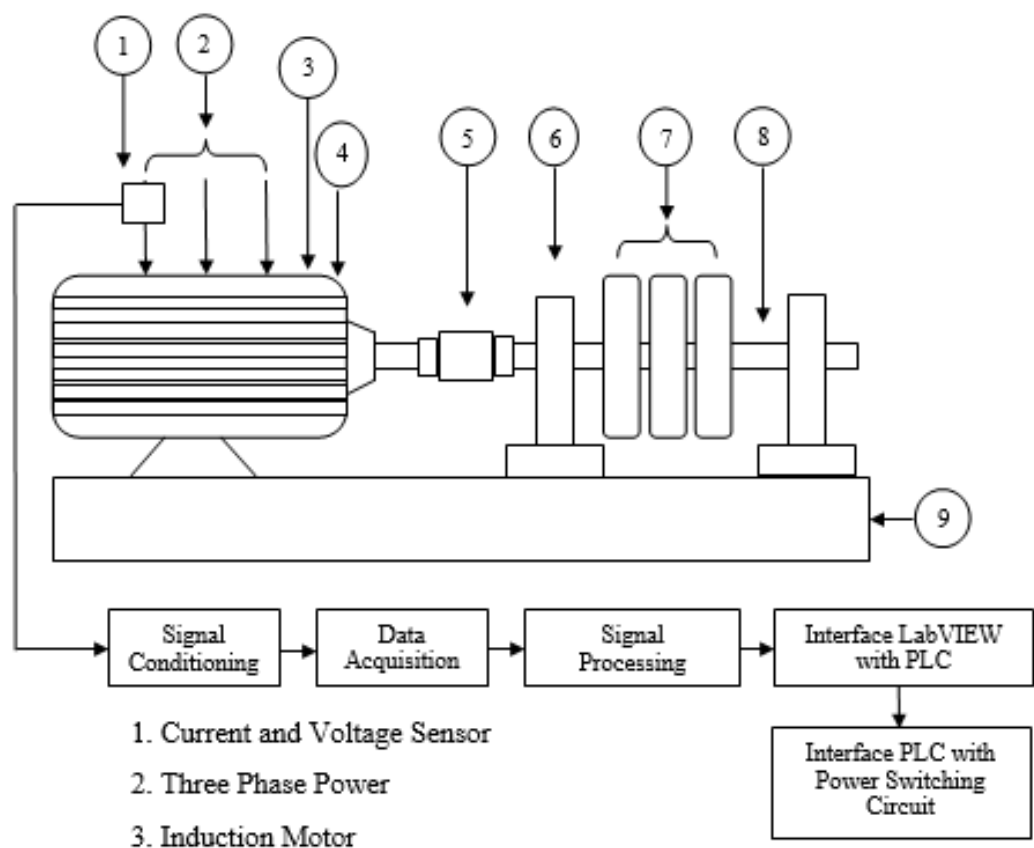

3. Induction Motor

4. Bearing under Test

5. Mechanical Coupling

6. Bracket

7. Bearing Load

8. Steel Shaft

9. Base

(d)

Fig. 1. The structure of the components used in the test set-up (a) bearing geometry (b) damaged cage (c) structure of steel plates used as load (d) complete hardware integration

Table III. Summary of Analysis of Motor Current and Power Data

\begin{tabular}{|c|c|c|c|c|c|c|}
\hline \multirow{2}{*}{ Load } & \multicolumn{4}{|c|}{ Analysis of Motor Current } & \multicolumn{3}{c|}{ Analysis of Motor Power } \\
\cline { 2 - 8 } & $\begin{array}{c}\text { Fault Frequencies } \\
(\mathrm{Hz})\end{array}$ & $\begin{array}{c}\text { Amplitude for } \\
\text { Healthy Cage (dB) }\end{array}$ & $\begin{array}{c}\text { Amplitude for } \\
\text { Faulty Cage (dB) }\end{array}$ & $\begin{array}{c}\text { Fault Frequencies } \\
(\mathrm{Hz})\end{array}$ & $\begin{array}{c}\text { Amplitude for } \\
\text { Healthy Cage (dB) }\end{array}$ & $\begin{array}{c}\text { Amplitude for } \\
\text { Faulty Cage (dB) }\end{array}$ \\
\hline No Load & $40.60,59.40$ & $-58.25,-55.61$ & $-55.39,-52.30$ & $90.60,109.40$ & $-54.28,-51.86$ & $-46.90,-44.45$ \\
\hline Medium Load & $40.80,59.20$ & $-62.15,-58.79$ & $-57.19,-53.54$ & $90.80,109.20$ & $-56.54,-53.69$ & $-46.69,-42.42$ \\
\hline Full Load & $41.20,58.80$ & $-57.74,-54.21$ & $-49.26,-46.81$ & $91.20,108.80$ & $-54.73,-50.92$ & $-39.56,-34.68$ \\
\hline
\end{tabular}




\section{Results and Discussions}

A. Case Study 1: Bearing cage analysis using motor power data

The data for motor power has been obtained from the voltage and current sensors and has been plotted in frequency domain. The results for the healthy and faulty cage for no-load and full load conditions are shown in Figure 2 and 3 respectively. A change in amplitude values has been observed from healthy cage and faulty cage graphs. The change in amplitude is minor for no-load condition. However, a larger change in amplitude has been observed for full load condition. This change in amplitude is used as an indicator of appearance of fault in cage.

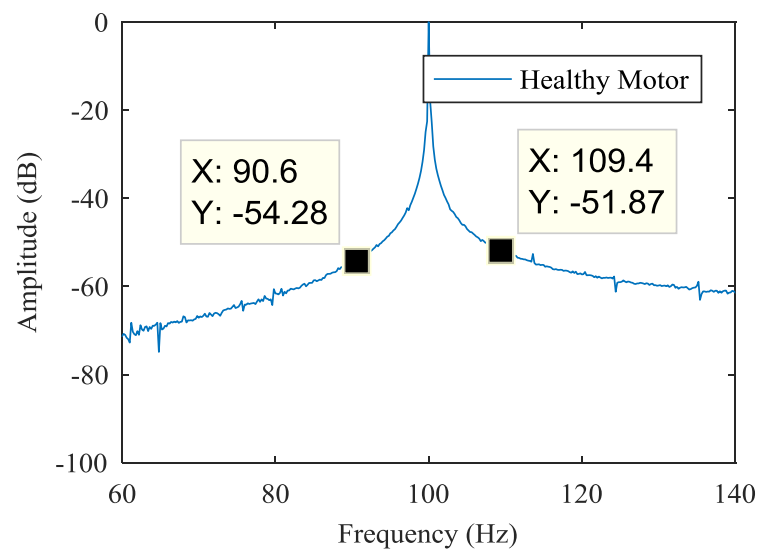

(a)

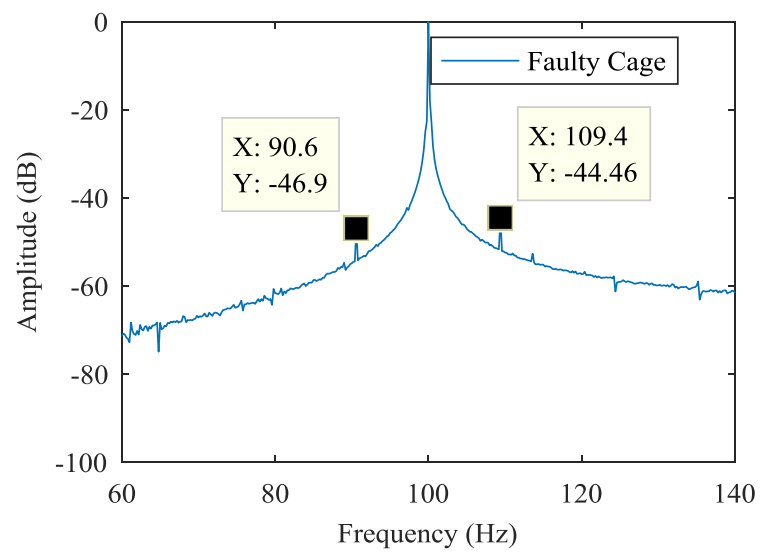

(b)

Fig. 2.The plot of motor power under no load for (a) healthy cage (b) faulty cage

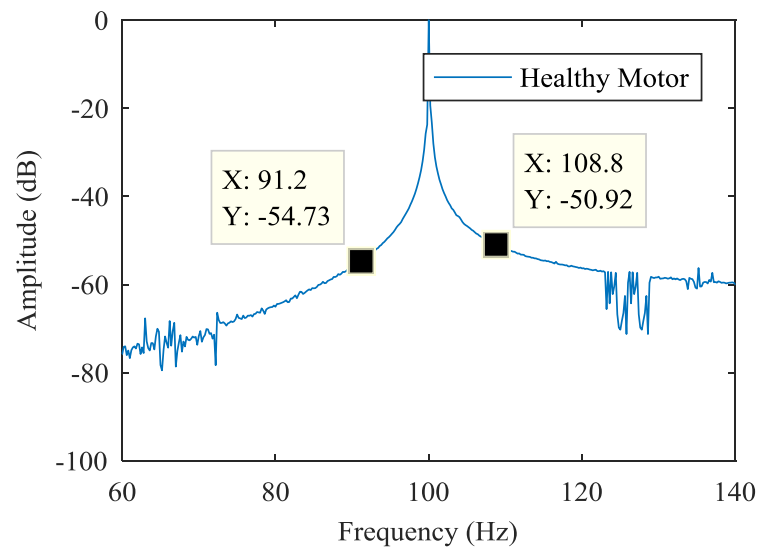

(a)

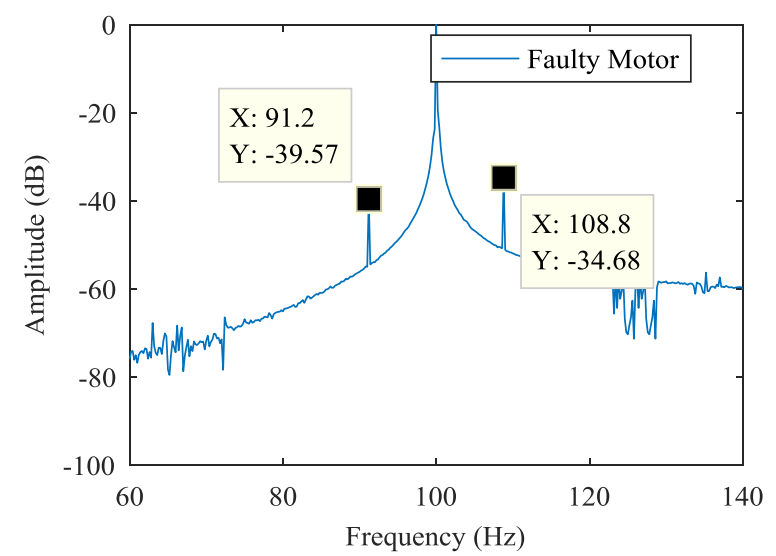

(b)

Fig. 3.The plot of motor power under full load for (a) healthy cage (b) faulty cage

B. Case study 2: Bearing cage analysis using motor current data

The motor current data has been collected through the current sensor and has been plotted in frequency domain. The results for the healthy cage and faulty cage for no-load and full-load conditions are shown in Figure 4 and 5. A small change of $3 \mathrm{~dB}$ has been observed under no-load condition but a comparatively larger change in amplitude has been observed for full load condition. 


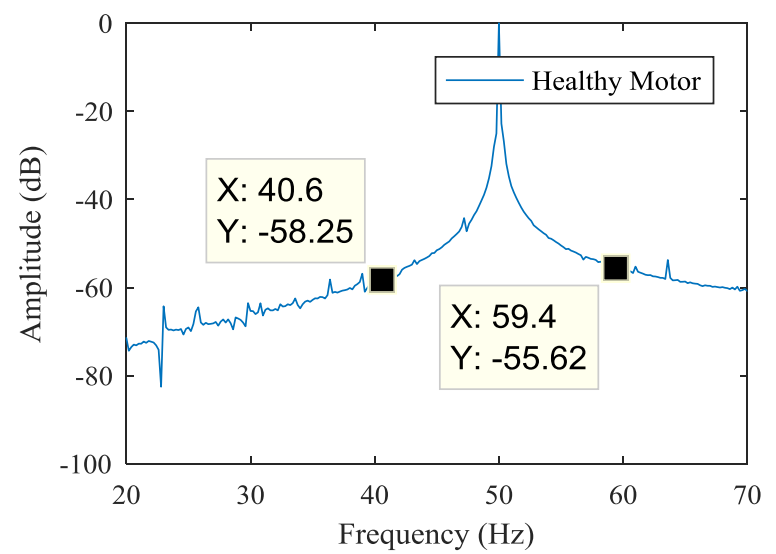

(a)

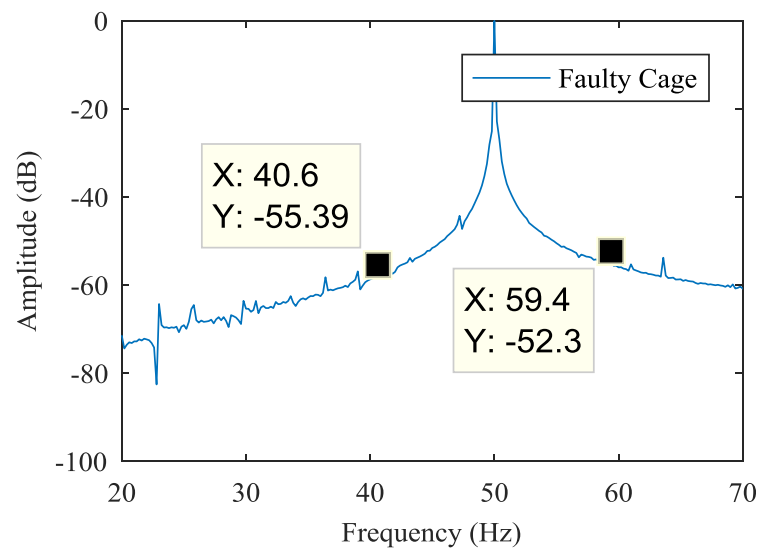

(b)

Fig. 4.The plot of motor current data under no load for of (a) healthy cage (b) faulty cage

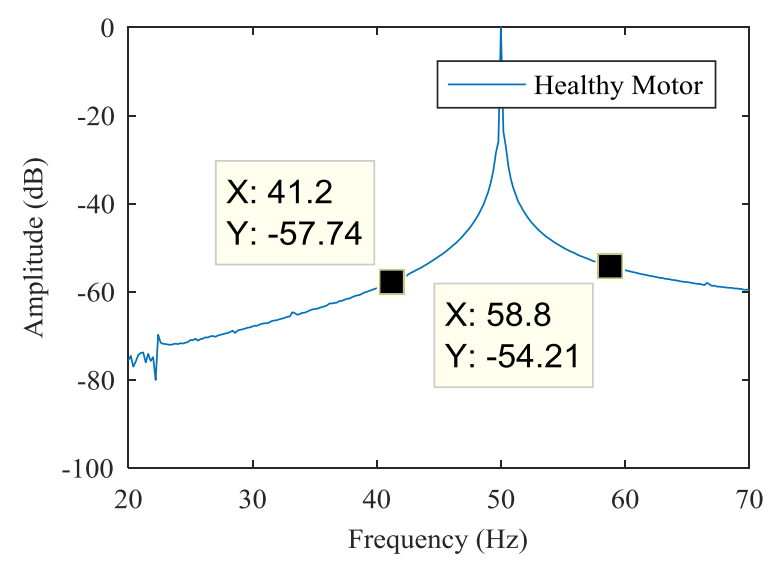

(a)

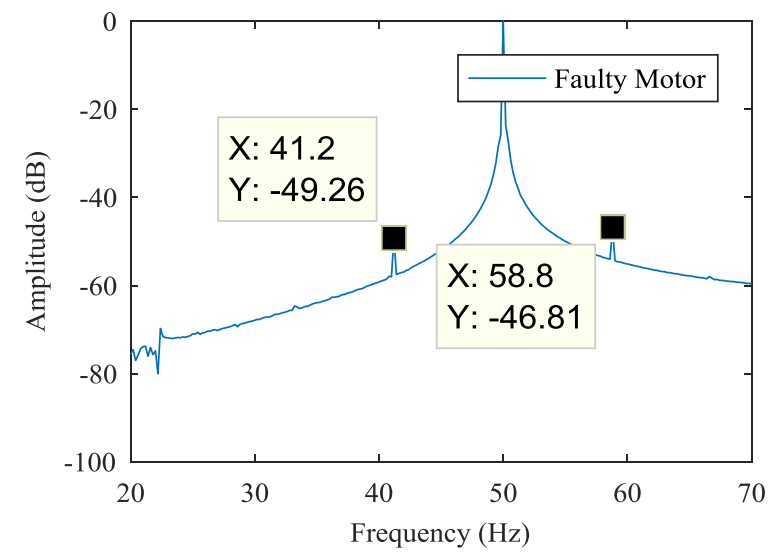

(b)

Fig. 5.The plot of motor current data under no load for of (a) healthy cage (b) faulty cage

The comparison of motor power analysis and current analysis has been summarized in Table III. The comparison indicates that power analysis contains stronger fault signatures as they have higher change in amplitude values as compared to motor current analysis. Thus, motor power analysis is a more suitable option for the online diagnostics and classification system for higher reliability of true detection.

\section{Conclusions}

Development of condition monitoring methods to improve the reliability and to reduce the maintenance equipment cost is an active area of research. This paper has presented a meaningful comparison of two non-intrusive and cost-effective diagnosis methods known as motor current analysis and power analysis methods. The diagnosing ability of both methods have been tested in the analysis of bearing cage faults. The experimental results have concluded that both non-intrusive methods possess ability to diagnose cage faults. However, the power analysis method provides amplified amplitudes at cage fault frequency. Thus, fault analysis and classification is more easy using power analysis technique.

\section{Acknowledgement}

The authors acknowledge the Najran University Saudi Arabia and Universiti Teknologi PETRONAS, Malaysia for providing research facilities and technical support.

\section{References}

[1] Muhammad Irfan, Nordin Saad, Rosdiazli Ibrahim, Vijanth S Asirvadam and Muawia Magzoub, "An Online Fault Diagnosis System for Induction Motors via Instantaneous Power Analysis," Tribology Transactions, Vol. 60, No. 4, pp. 592-604, July 2017. 
[2] A. Glowacz, W. Glowacz, Z. Glowacz, J. Kozik, M. Gutten, D. Korenciak, Z. F. Khan, M. Irfan and E. Carletti, "Fault Diagnosis of Three Phase Induction Motor using Current Signal, MSAF-Ratio15 and Selected Classifiers," Archives of Metallurgy and Materials, Vol. 62, No. 4, pp. 2413-2419, December 2017.

[3] Muhammad Irfan, Nordin Saad, Rosdiazli Ibrahim, Vijanth S Asirvadam, Alwadie A and Muhammad Aman, "Analysis of Distributed Faults in Inner and Outer Race of Bearing via Park Vector Analysis Method," Neural Computing \& Applications, May 2017.

[4] M. Aman, Nursyarizal, Tayyab, and Muhammad Irfan and Nordin Saad "An Intelligent Automated Method to Diagnose and Segregate Induction Motor Faults," Journal of Electrical Systems, June 2017.

[5] M. Aman, Nursyarizal, Tayyab, and Muhammad Irfan, "An Unsupervised On-Line Method to Diagnose Unbalanced Voltage in Three-Phase Induction Motor," Neural Computing \& Applications, April 2017.

[6] P. J. Tavner, L. Ran, J. Pennman, and H. Sedding "Condition Monitoring Of Rotating Electrical Machines," Letchworth, England: Research Studies Press Ltd. 2008.

[7] Fatigue and fracture, ASM Handbook, Vol. 19, ASM International, 1996.

[8] P. Zhang, Y. Du, T. G. Habetler, and B. Lu, "A Survey Of Condition Monitoring And Protection Methods for Medium-Voltage Induction Motors," IEEE Transactions on Industry Applications, Vol. 47, No. 1, pp. 34-46, January 2011.

[9] Zhiwei Gao, Carlo Cecati and Steven X. Ding, "A Survey Of Fault Diagnosis And Fault-Tolerant Techniques Part I: Fault Diagnosis with Model Based and Signal-Based Approaches," IEEE Transactions on Industrial Electronics, 2015

[10] Z. Hameed, Y.S. Hong, Y.M. Cho, S.H. Ahn, C.K. Song, "Condition Monitoring And Fault Detection Of Wind Turbines and Related Algorithms: A Review," Renewable and Sustainable Energy Reviews, Vol. 13, pp. 1-39, 2009.

[11] F. Immovilli, A. Bellini, R. Rubini, and C. Tassoni, "Diagnosis of Bearing Faults In Induction Machines by Vibration or Current Signals: A Critical Comparison," IEEE Transactions on Industry Applications, Vol. 46, No. 4, pp. 1350-1359, July 2010

[12] L. Navarro, M. Delgado, J. Urresty, J. Cusidó and L. Romeral, "Condition Monitoring System for Characterization of Electric Motor Ball Bearings with Distributed Fault Using Fuzzy Inference Tools," IEEE Instrumentation and Measurement Technology Conference (I2MTC), Austin , May, 2010.

[13] Aiwina Heng, Sheng Zhang, Andy C.C. Tan, Joseph Mathew, "Rotating Machinery Prognostics: State of the Art, Challenges and OpportunitiesA Review," Mechanical Systems and Signal Processing, Vol. 23, pp. 724-739, 2009.

[14] Zulma Yadira Medrano Hurtado, Carlos Perez Tello and Julio Gomez Sarduy, "A Review on Detection and Fault Diagnosis in Induction Machines," Publicaciones en Ciencias y Tecnologa, Vol. 8, No.01, July 2014.

[15] S. B. Alegranzi, J. F. Gonçalves and H. M. Gomes, "Ball Bearing Vibration Monitoring for Fault Detection by the Envelope Technique," Blucher Mechanical Engineering Proceedings, Vol. 1, Issue 1, May 2014.

[16] Ehsan Tarkesh Esfahani, Shaocheng Wang, and V. Sundararajan, "Multisensor Wireless System for Eccentricity and Bearing Fault Detection in Induction Motors," IEEE/ASME Transactions on
Mechatronics, Vol. 19, No. 3, June 2014.V.N. Patel, N. Tandon and R.K. Pandey, "Defect Detection in Deep Groove Ball Bearing in Presence of External Vibration using Envelope Analysis and Duffing Oscillator," Measurement, Vol. 45, pp. 960-970, 2012.

[17] V.N. Patel, N. Tandon and R.K. Pandey, "Vibrations Generated by Rolling Element Bearings having Multiple Local Defects on Races," Procedia Technology, Vol. 14, pp. 312-319, 2014.

[18] Sukhjeet Singh, Amit Kumar and Navin Kumar, "Motor Current Signature Analysis for Bearing Fault Detection in Mechanical Systems," Procedia Materials Science, Vol. 6, pp. 171-177, 2014.

[19] Muhammad Irfan, Nordin Saad, Rosdiazli Ibrahim and Vijanth S Asirvadam, " Diagnosis of Distributed Faults in Outer Race of Bearings via Park's Transformation Method," The 10th Asian Control Conference (ASCC) Kota Kinabalu, Malaysia, June 2015.

[20] Muhammad Irfan, Nordin Saad, Rosdiazli Ibrahim and Vijanth S Asirvadam, "A Non Invasive Fault Diagnosis System for Induction Motors in Noisy Environment," IEEE International Conference on Power and Energy (PECon), Malaysia, December 2014.

[21] Muhammad Irfan, Nordin Saad, Rosdiazli Ibrahim and Vijanth S Asirvadam, "Analysis of Bearing Outer Race Defects in Induction Motors," The 5th IEEE International Conference on Intelligent and Advanced Systems (ICIAS), Malaysia, June 2014.

[22] Muhammad Irfan, Nordin Saad, Rosdiazli Ibrahim and Vijanth S Asirvadam, "An Approach to Diagnose Inner Race Surface Roughness Faults in Bearings of Induction Motors," IEEE International Conference on Signal and Image Analysis (ICSIPA), Kuala Lumpur, Malaysia, October 2015.

[23] Muhammad Irfan, Nordin Saad, Rosdiazli Ibrahim, Vijanth S Asirvadam, Alwadie A and Muhammad Aman, "An Assessment on the Non-Invasive Methods for Condition Monitoring of Induction Motors," Fault Diagnosis and Detection- ISBN 978-953-51-5011-4, InTech Publishing, May 2017.

[24] Muhammad Irfan, Nordin Saad, Rosdiazli Ibrahim and Vijanth S Asirvadam, "Analysis of Bearing Surface Roughness Defects in Induction Motors," Journal of Failure Analysis and Prevention, Vol. 15, No. 5, pp. 730-736, August 2015.

[25] Muhammad Irfan, Nordin Saad, Rosdiazli Ibrahim and Vijanth S Asirvadam, "Condition Monitoring of Induction Motors via Instantaneous Power Analysis," Journal of Intelligent Manufacturing, Volume 28, Issue 6, pp 1259-1267, August 2017.

[26] Muhammad Irfan, Nordin Saad, Rosdiazli Ibrahim and Vijanth S Asirvadam, "An Intelligent Diagnostic Condition Monitoring System for AC Motors via Instantaneous Power Analysis," International Review of Electrical Engineering, Vol.8, No. 2, pp. 664-672, April 2013. 